\title{
Assurance of quality, safety and efficacy of vaccines through compliance of quality management
} processes

\begin{abstract}
Vaccines develop the immune response against disease and are the intricate biological entities containing one or more than one antigen beside an adjuvant. In order to ensure that manufactured vaccines are safe, potent, and of consistent quality, four primary controls of bio safety namely: PPE; facility design; SOPs and leadership are required at every step of manufacture and quality control testing until the administration of vaccine to the target population. The safety and efficacy of testing methods are based upon the data validation of several years. With time the advancements in vaccine production techniques has brought about advancements in testing techniques as well. These improvements have enabled scientists to detect highly sensitive potential hazards which were a dream few years back. An increasing assemblage of physico-chemical methods permits much better characterization of the product. In addition to advance tests vaccine regulations necessitate quite a few numbers of strategies to ensure the safety and potency of the vaccine/serum. These measures include cell banking, seed lot system, supplier audits, master seed, compliance with good manufacturing practices, independent lot release from National Regulatory Authority and increased (pre and post) marketing surveillance to look for possible adverse effects following immunization (AEFI). Together all these methods ensure the quality of vaccine for the purpose it is manufactured against.
\end{abstract}

Volume 8 Issue 5- 2019

\author{
Syeda Shazia Adeel,' Muhammad Babar Taj,'2 \\ Muhammad Wasif Malik, ${ }^{3}$ Amina Najam, ${ }^{4}$ \\ Kanwal Batool' \\ 'Department of Quality Assurance, National Institute of Health, \\ Pakistan \\ ${ }^{2}$ Department of Chemistry, Islamia University of Bahawalpur, \\ Pakistan \\ ${ }^{3}$ Department of field epidemiology and disease surveillance \\ division, National Institute of Health, Pakistan \\ ${ }^{4}$ Department of quality Control Laboratory, National Institute \\ of Health, Pakistan
}

\begin{abstract}
Correspondence: Muhammad Babar Taj, Department of Chemistry, Islamia University of Bahawalpur 63100, Pakistan, Te +923007542669, Email drbabartaj@gmail.com
\end{abstract}

Received: September 28, 2019 | Published: October 28, 2019

Keywords: quality assurance, quality control, drug evaluation, methods and trends, vaccine monitoring legislations, vaccine standards, good manufacturing practices, safety of vaccine, validations, consistency of production, toxoids, genetic markers

Abbreviations: NRA, national regulatory authority; PERT, product enhanced reverse transcriptase; TSE, transmissible spongiform encephalopathies; CJD, creutzfeldt-Jakob disease; PMS, post marketing surveillance; BSE, bovine spongiform encephalopathy

\section{Introduction}

Immunization is one of the most rewarding effective and economical health interventions known to mankind to-date. Since last 50 years successful vaccination campaigns has resulted in extermination of small pox, dropped global polio incidence by $99 \%$ and had efficaciously reduced the disability, illness and morbidity caused by the vaccine preventable diseases. Immunization is a crucial constituent behind the efforts taken to meet millennium development goals reducing the mortality in children under-five by over 50 percent, decreasing from 90 to 43 deaths per 1,000 live births between 1990 and 2015. ${ }^{1}$ To ensure continuous positive results from immunization WHO has stressed upon the need of stringent quality assurance measures which is dependent upon control / monitoring of raw materials; adequacy of production processes, suitability quality control methods and specifications, and control / monitoring of final finished products. The present paper assesses all the essential tools required for the development of a quality biological product. These factors safeguard the safety, efficacy, integrity and quality of vaccines; a result of adequate quality management system which is based upon quality risk management approach (validations, consistency of production, vendor qualifications, independent lot release etc).

\section{Efficacy of vaccines}

At present, approved vaccines are present worldwide to prevent the human infectious diseases against more than 35 microbes. Although definite number of products is significantly high, as many being combination vaccines and formulations intended for different age groups, different regions and both private and public sector markets. ${ }^{2}$ Conventional vaccines having edge of being in use since several decades, use bioassay for production and control testing before the final release of the product, in these vaccines animal-based potency testing is required to facilitate the production of consistent, safe and potent every single manufactured lot. Tetanus and diphtheria toxoids, cell culture rabies vaccine are few that fall in this category. Effectiveness and safety of vaccines is determined by the immense experience in the field via post marketing monitoring of adverse effects and reduction in cases. For few of the above mentioned vaccines serological markers are also available. As for example tetanus antitoxin's protective role is known. These titers were developed after animal model studies by developing the correlation between antibody tiers and severity of tetanus. The minimum protective titer is $0.01 \mathrm{IU} / \mathrm{ml}$ serum but there are cases of tetanus in persons having $0.16 \mathrm{IU} / \mathrm{ml}$ titer. ${ }^{3,4}$ For diphtheria the protective titer level is $0.01 \mathrm{IU} / \mathrm{ml}$ serum but better protection is provided in individuals with more than $0.09 \mathrm{IU} / \mathrm{ml}$ titer. ${ }^{5}$ In case of cell culture rabies vaccine, WHO recommends antibody titer of more than $0.05 \mathrm{IU} / \mathrm{ml}$ as proper immune response. ${ }^{6}$

Through different regulations it is strongly emphasized that the 
testing procedures for vaccines produced from human or animal cell substrates must ensure the absence of contaminating viruses that cause disease in humans. For measles vaccine which is produced in chick embryos a substantial amount of testing has been performed to ensure the absence of contaminating adventitious agents. Additionally, all the eggs used for preparation of master seed lot have to be specific pathogen i.e. free from 32 different diseases including avian leucosis. Although this retro virus is not pathogenic to humans but it is considered important to be free from retro viruses.

\section{Developments in vaccine testing}

With development in molecular and genetic biology, advance techniques for physicochemical analysis, characterization and purification of proteins, polysaccharides antigen and use of PCR amplification has greatly enhanced the capacity for detection of even traces of virus contaminants. These techniques are sensitive to such level that they can detect even part of viral genomics. Product enhanced reverse transcriptase (PERT) assay is another technique which is used for detection of even minute traces of reverse transcriptase in bulk or final product of chick cell origin attenuated vaccines. Ongoing research has proved that the probability of whether the presence of a vaccine virus itself (e.g., measles, rubella, yellow fever) in chick cells could host the restoration of retroviral infectivity as being exceedingly small. Nevertheless extra analysis is required to rectify these hypothetical concerns. ${ }^{8,9}$

\section{Limitations of vaccine testing in traditional methods}

Traditional methods have limitations in context of risk assessment and management like agents of transmissible spongiform encephalopathy's (TSE) of bovine origin (BSE) which have new variant i.e. Creutzfeldt-Jakob disease (CJD) is one of the limitations of the traditional method. To handle such limitations different approach for vaccine safety are necessary. The measures many include: to avoid the use of bovine origin materials wherever possible; If its necessity then use of low risk tissues should be given preference; or supply order given to countries where zero reports for BSE and must have compulsory BSE reporting system. ${ }^{10}$ Efforts are on way to standardize the assays. ${ }^{11}$

\section{Additional procedures to ensure safety and quality of vaccines}

This is a proven fact now that quality and safety of vaccines is dependent on several fundamental principles like use of properly characterized homogenous starting materials of known origin and source along with certificate that supplier is carrying out regular validations. Validation and revalidation of production and control testing starting from raw materials to intermediate and final product, intermediate and final testing, labeling, packaging and supply process. Proof of consistency in production as per approval of National Regulatory Authority (NRA); Independent lot release of each batch of product from National Regulatory authority, Pre and post marketing surveys to show the safety and efficacy of target vaccine, the first three conditions can only be met by strictly following the good manufacturing practices. ${ }^{12}$

\section{Characterization of starting materials}

Apart from quality control testing of each component supplier audits also play a vital role in selection of qualified supplier.
Ensuring verification and validation of each and every production and analysis constituent is an essential function of quality assurance. Production systems based on cell lines, or cell banks provide adequate characterization of raw material. Quality assurance ensures that production system must be based on well characterized master and working cell bank system. Few examples of characteristics to be considered are: viability during storage, genealogy, genetic markers, tumorigenicity, absence of detectable contaminants etc. This characterization involves the measure of growth characteristics at every stage of cell life span. Including potential loss in efficacy of cell during storage conditions. ${ }^{12,13}$ Traditional sterility testing method is used for the detection of potential fungal and bacterial presence in product. Presence of mycoplasma is detected by combination of traditional culture method and in-vitro testing. There are essentially four sources of viral contamination: infected source material, Hybridomas, contaminated biological reagents and contamination during processing. Required tests for detection of these four contaminations are electron microscopy, detection of reverse transcriptase, in vivo and in vitro infectivity tests, induction of antibody response in laboratory animals of different species, and/or tests specific for humans. ${ }^{12,13}$

\section{Validation of production process}

Regular, periodic validation that manufactured product is safe potent and each batch is of equally high quality is another major function of assuring the quality and safety of vaccine. Validation is the gathering and analysis if facts, launch at process evaluation stage and resumed throughout the phases of production which ensures that manufacturing process that contains equipment, building, personnel and materials which have the ability of getting the expected results constantly and continuously. Validation is carried out through review and evaluation of whole system systematically including facilities, equipment, and processes. It is focused to identify whether the whole system carried out their assigned functions satisfactorily and effectively without any interruption. Validation process has proved to demonstrate a high level of assurance that invariable batches of product will be produced, and they will all the criteria of NRA. Hence NRA will officially certify the finished product. ${ }^{14}$ Validation is helpful to the organization in many diverse ways like increase in understanding of the processes, increase in confidence regarding the product quality and safety, decrease in the risks of processing problems, and decrease in the risk of regulatory non-compliance. WHO defines three methods of validation which are prospective, concurrent and retrospective? These methods are used depending upon the processing conditions. The process is considered validated when every step of production and control testing is minimum consecutive thrice repeated under actual condition and it remains validated till specific time period with unchanged measured aspects. ${ }^{15}$ Revalidation is carried out if any deviations take place at any stage, equipment, method, facility of manufacturing or control testing. ${ }^{16}$

\section{Proof of consistency in production}

Validation at each and every step of production and control testing pave the way for consistency in production techniques and enhance the confidence of manufacturer on the product's quality. This means that the critical aspects of the vaccine product, which most of the time are measured through ongoing production procedures and by final product specifications, are consistently achieved for different product cycles. Product identification are set to measure to , the maximum thinkable extent, that the vaccine concerned will be without harm 
and effective when administrated to the targeted population. ${ }^{17}$ Safety and efficiency of vaccines are determined through human clinical trials. Once safety and efficiency have been certified for human, then it is necessary that the subsequent batches of product must show consistency with the vaccine lots used for clinical trials. Thus, final production tests become a proof of consistency for production cycles and ensure that every single batch of vaccine has same features as a batch which was proved to be safe and effective in the clinical trials. ${ }^{18}$

\section{Independent lot release}

WHO insists on the formation of NRA in every country for independent lot release of every batch of every vaccine being manufactured in that country either human or veterinary? This proves to be one of the key elements in assurance of vaccine quality. This lot release process provides a major check on manufacturer's performance in controlling Lot release by the NRA and is a key to the control of vaccines. It also delivers as a vital check on starting materials, the manufacturing process itself and analytical techniques in detail which are claimed by the manufacturer. The minimum lot release should be evaluated on review of summary of lot protocols, which contain facts of the particular lot in addition some or all laboratory testing that NRA can carry out. The responsibility for lot release rests with authorized head of NRA. ${ }^{19,20}$

\section{Post marketing surveillance (PMS)}

Another major instrument for overseeing vaccine quality and efficiency is post marketing surveillance (PMS). Although unusual but sometimes harmful or adverse events follow administration of vaccines, very few of these occurrences are caused because of intrinsic features of vaccine products. Careful attention must be given to those events which were not noticed during pre-licensing clinical trials mainly because of sample size of the vaccinated population. Some serious events have been reported which were mainly the result of programmatic errors like improper handling and inadequate maintenance of vaccine cold chain or improper vaccine administration practices. $^{21,22}$ PMS may allow observing of vaccine efficacy mainly by measuring decrease of cases. PMS can also provide conclusive evidence on effectiveness of various vaccine products. Therefore, it is inevitable to carry out PMS in a country. It is duty of NRA to monitor the safety and efficacy of vaccines products already in the market. However, country specific immunization programmed should also carry out case investigation studies to assess safety and efficacy of vaccine products used for mass immunization in that country. ${ }^{20,22,23}$ Even though national regulatory process includes examination and verification of clinical trials carried out to ensure safety and efficacy of vaccine products, but these can be complimented by PMS on larger target population. One such study was conducted in Ghana involving Health care practitioners. These days' regulatory bodies throughout the world are making it a part of compliance criterion for manufacturers to perform PMS as a further control on safety and efficacy of the vaccine. ${ }^{23}$

\section{Adverse events following immunization (AEFI)}

Although vaccines are associated with effective and strong prevention of against the deadly diseases but adverse reactions are also associated with them. Most of these reactions are insignificant but still they are of a global concern. Example Measles vaccine which is administered almost 100 million infants around the globe annually is associated with adverse effects like fever, rash, pain, anaphylaxis etc. WHO has made guidelines regarding handling of vaccines properly in order to minimize the AEFIs. AEFI is investigated depending upon the number of cases, nature of events, community perceptive etc. Criteria for investigation are decided by catering all these mentioned factors. These AEFI are majority of the time due to improper administration, inappropriate cold chain maintenance, incorrect reconstitution with diluents etc. As a result WHO has intensified the control measures on production, quality, safety and efficacy of vaccine along with pre and post marketing surveillance but also established protocols for the training of health care professionals including physicians, nurses, vaccinators, technicians who are involved in administration of vaccine to the end user. These training protocols cater identification, prevention and treatment of AEFI. ${ }^{21,24,25}$

\section{New vaccines: an increasingly complex situation}

With introduction of new combination vaccines in market the assurance of vaccine quality has become more complex and cumbersome process. As a result, NRA has increased the regulatory requirements for the manufacturers to meet the safety and efficacy criterions. HIB, D Tap, DTP, MMRV, DTP/IPV, DTP-HEP B, DTPHEP B-HIB are some of the examples of the combination vaccines. Vaccines are produced against same disease, but they have different manufacturing components or processes (formulations composition, concentrations, antigenic structures etc example DTP-HEP B, and DTP-HEP B-HIB) thus making those separate vaccines and consequently NRA treat them as different vaccines. ${ }^{26}$

\section{Conclusion}

This article summarizes that highly sensitive tests for evaluation and verification of safety and quality of vaccines are available. Use of these technological advance techniques along with strict compliance of regulatory measures laid down by NRA can ensure that the manufactured vaccines are safer than ever before. Field investigation also supports these statements. There is, however a need to study what to do with and how to utilize the data obtained from innovative and refined analytical technologies. This review also emphasizes that for the process of vaccine development, vaccine safety profile, key test parameters correlating with clinical demonstration must be considered. It also highlights that NRA must take multi-faceted approach in sustaining and guaranteeing the safety, efficacy and consistency of the manufactured vaccines along with laborious post marketing surveillance activities.

\section{Acknowledgments}

None.

\section{Conflicts of interest}

The author declares that there are no conflicts of interest.

\section{Funding}

None.

\section{References}

1. Malcolm Molvneux, Elizbeth Molvneux. Reaching millennium development goal 4. The Lancet Comment. 2016;4(3); E146-E147. 
2. Immunization, vaccines and Biologicals. 2017.

3. Immunological basis of immunization. Series Module 3:Tetanus. WHO 2018;1-61.

4. Passen EL, Andersen BR. Clinical tetanus despite a 'Protective" level of toxin - neutralizing antibody. JAMA. 1986;225(9):1171-1173.

5. Immunological basis of immunization. Series Module 2: Diptheria. WHO. 2009;1-39.

6. Immunological basis of immunization. Series Module 17: Rabies. WHO. 2017;1-49.

7. GMP's 21 CFR part 821-Quality system regulations. 2018.

8. Pyra h, Boni J, Scupbach J. Ultrasensitive retrovirus detection by a reverse transcriptase assay based on product enhancement. Proc Natl Acad Sci USA. 1994;91(4):1544-1548.

9. Boni J, Stalder J, Reigel F, et al. Detection of reverse transcriptase activity in live attenuated virus vaccines. Clinical Diagnosis and Virology. 1996;5(1):43-50.

10. Report of WHO Consultation on medicinal and other products in relation to human and animal transmissible spongiform encephalopathy, Geneva, Switzerland:WHO. 1997.

11. Asher DM, Podilla AM, Pocchiari M. WHO consultation on diagnostics procedures for transmissible encephalopathies, need for reference reagents and reference panels. Biologicals. 1999;27(3):263-270

12. Ahmed MMF, Alitamsar H, Angtrakool P, et al. Good Manufacturing Practices for biological products. WHO Technical Report Series No. 999. 2016;Annex 2:104-130.

13. Grachev V, Magrath D, Griffiths E. WHO requirements for the use of animal cells as in vitro substrates for the production of biologicals (Requirements for biological susbstances no. 50). Biologicals. 1998;26(3):175-193.

14. US FDA-Guidance for Industry (draft): Analytical Procedures and Methods Validation for Drugs and Biologics, 2015.
15. EU GMP, Volume 4, Chapter 6: Quality Control. 2014.

16. ICH Q2(R1): Validation of Analytical Procedures: Definitions and Methodology, Geneva: EMEA. 2006;1-15.

17. Sneha Mary Abraham, N Vishal Gupta. Importance of Quality Metrics: A Review. IJPQA. 2016;7(1):15-16.

18. WHO good manufacturing practices for biological products. Technical Report Series 999. 2016.

19. William C Lamanna, Johann Holzmann, Hillel P Cohen, et al. Maintaining consistent quality and clinical performance of biopharmaceuticals. Expert Opin Biol Ther. 2018;18(4):369-379.

20. Guidelines for Independent Lot Release of Vaccines by Regulatory Authorities, Adopted by the WHO Expert Committee on Biological Standardization. 2010.

21. World Health Organization WHO Vaccine Safety: Adverse Events and Vaccine Reaction. 2018.

22. Adriana Parella. Post marketing vaccine safety passive surveillance: an exploratory study of parent and health care provider reporting of adverse events following immunization (AEFI). 2014.

23. Netterlid Eva, Mansson ME, Hakansson A. Surveillance of vaccine safety: comparison of parental reports with routine surveillance and a clinical trial. Vaccine. 2009;27(14):2042-2047.

24. Petar Yamoah, Varsha Bangalee and Fraisa Oosthuizen. Knowledge and perceptions of adverse events following immunization among healthcare professionals in Africa: a case study from Ghana. Vaccines (Basel). 2019;7(1):28.

25. Causality assessment of an adverse event following immunization (AEFI). User manual for the revised WHO classification. 2013.

26. Recommendations for production and control of Haemophilus Influenza type b conjugate vaccines. TRS. 897, 2000. 